\title{
The reported intakes of sugar sweetened beverages by 10-11 year old children participating in the CHANGE! healthy eating intervention
}

\author{
G.L. Stone ${ }^{1}$, I.G. Davies ${ }^{2}$, R. Dagger ${ }^{3}$, K. Mackintosh ${ }^{4}$, S.J. Fairclough ${ }^{5}$ and J.C. Abayomi ${ }^{2}$ \\ ${ }^{1}$ Faculty of Health and Social Care, Edge Hill University, St Helens Road, Ormskirk, L39 4QP., ${ }^{2}$ Faculty of
} Education, Health and Community, Liverpool John Moores University, Barkhill Road, Liverpool L17 6BD., ${ }^{3}$ Faculty of Science and Social Science, Liverpool Hope University, Liverpool L16 9JD., ${ }^{4}$ Applied Sports Technology Exercise and Medicine Research Centre, College of Engineering, Swansea University, Swansea SA2 8PP. and ${ }^{5}$ Faculty of Arts and Sciences, Edge Hill University, St Helens Road, Ormskirk, L39 4QP

Free sugars in foods and particularly sugar sweetened beverages (SSB) have increasingly, and somewhat controversially, been the focus of public health concerns ${ }^{(1)}$. SSB are considered to form part of an 'unhealthy diet' which with other dietary and lifestyle factors, contribute to total disease burden in England ${ }^{(2)}$. According to the National Diet and Nutrition Survey ${ }^{(3)}$ 'non-alcoholic beverages' contributed 27-30\% of non-milk extrinsic sugars (NMES) for children aged $<10$ years old. A 20-week teaching curriculum was developed for The CHANGE! Project (Children's Health, Activity, and Nutrition: Get Educated!) and aimed to promote the benefits of healthy eating, including a reduction in SSB intakes, by making small behavioural changes. This curriculum was delivered by teachers to Year 6 children $(n=290$ total; $n=138$ intervention; $n=152$ control) in the intervention schools in Wigan, following completion of the baseline (BL) data collection.

The participants completed a validated 24-hour recall food intake questionnaire ${ }^{(4)}$ at BL and post-intervention (PI). There were no significant differences in each groups' reported intakes of SSB, between BL or PI ( $p \geqslant 0 \cdot 069)$, or between the groups at BL and PI ( $p \geqslant$ $0 \cdot 371$ ). There was also a trend towards increased intakes of fizzy drinks and diet fizzy drinks for the intervention group between BL and PI. Cordial drinks appeared to be the most popular type of SSB. (Figure 1).

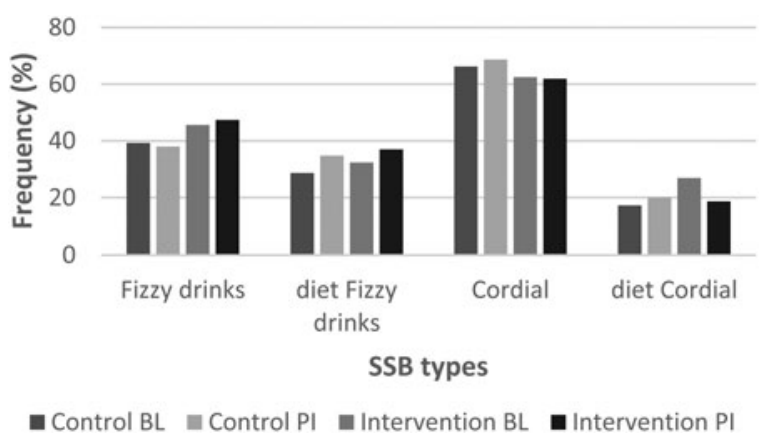

Fig. 1. frequencies of the reported intakes of $S S B$.

These results demonstrate that solely educating children about how to make changes to their beverage consumption habits does not automatically lead to behaviour change. Alongside the issues of age-related recollection bias and inaccurate recall of food intakes ${ }^{(5)}$, children's eating habits and behaviours are typically influenced by those of their parents and carers ${ }^{(6)}$. Involving the family as part of the education process could help to encourage more positive food choices. The recently announced levy on SSB for the UK, is anticipated to help towards reducing sales and consumption of these types of drinks. The effect of targeting one food source to reduce contribution to disease burden is debateable, however, the association between SSB and health issues is a complex one, so a multifactorial approach to improving children's food choices is essential.

1. Newens KJ, Walton J. A review of sugar consumption from nationally representative dietary surveys across the world. J Hum Nutr Diet 2016 ; 29 (2):225-240

2. Newton JN, Briggs ADM, Murray CJL, Dicker D, Foreman KJ, Wang H, et al. Changes in health in England, with analysis by English regions and areas of deprivation, 1990-2013: a systematic analysis for the Global Burden of Disease Study 2013. Lancet 2015 December 5;386(10010):22572274

3. Public Health England. National Diet and Nutrition Survey: Results from years 1, 2, 3, and 4 (combined) of the Rolling Programme (2008/20092011/2012). 2014; Available at: www.gov.uk/government/uploads/system/uploads/attachment_data/file/310995/NDNS_Y1_to_4_UK_report.pdf.

4. Johnson B, Hackett A, Roundfield M, Coufopoulos A. An investigation of the validity and reliability of a food intake questionnaire. J Hum Nutr Diet 2001; 14(6):457-465.

5. Burrows TL. A systematic review of the validity of dietary assessment methods in children when compared with the method of doubly labeled water J Am Diet Assoc 2010; 110(10):1501-1510.

6. Scaglioni S, Salvioni M, Galimberti C. Influence of parental attitudes in the development of children eating behaviour. Br J Nutr 2008; 99(S1):S25. 